\title{
BMJ Open What is the risk of prostate cancer mortality following negative systematic TRUS-guided biopsies? A systematic review
}

\author{
Sandra Miriam Kawa (D), Signe Benzon Larsen (D), John Thomas Helgstrand (D), \\ Peter Iversen, Klaus Brasso (i) , Martin Andreas Røder (i)
}

To cite: Kawa SM, Benzon Larsen S, Helgstrand JT, et al. What is the risk of prostate cancer mortality following negative systematic TRUSguided biopsies? A systematic review. BMJ Open 2020;10:e040965. doi:10.1136/ bmjopen-2020-040965

- Prepublication history and additional material for this paper are available online. To view these files, please visit the journal online (http://dx.doi org/10.1136/bmjopen-2020040965).

Received 27 May 2020 Revised 04 December 2020 Accepted 09 December 2020

Check for updates

(C) Author(s) (or their employer(s)) 2020. Re-use permitted under CC BY-NC. No commercial re-use. See rights and permissions. Published by BMJ.

Copenhagen Prostate Cancer Center, Urological Department, Rigshospitalet, Copenhagen, Denmark

Correspondence to Dr Sandra Miriam Kawa; sandramiriamkawa@gmail.com

\section{ABSTRACT}

Objective To investigate the risk of prostate cancerspecific mortality (PCSM) following initial negative systematic transrectal ultrasound-guided (TRUS) prostate biopsies.

Design Systematic review.

Data sources PubMed and Embase were searched using a string combination with keywords/Medical Subject Headings terms and free text in the search builder. Date of search was 13 April 2020.

Study selection Studies addressing PCSM following initial negative TRUS biopsies. Randomised controlled trials and population-based studies including men with initial negative TRUS biopsies reported in English from 1990 until present were included.

Data extraction Data extraction was done using a predefined form by two authors independently and compared with confirm data; risk of bias was assessed using the Newcastle-0ttawa Scale for cohort studies when applicable.

Results Four eligible studies were identified. Outcomes were reported differently in the studies as both cumulative incidence and Kaplan-Meier estimates have been used. Regardless of the study differences, all studies reported low estimated incidence of PCSM of $1.8 \%-5.2 \%$ in men with negative TRUS biopsies during the following 10-20 years. Main limitation in all studies was limited follow-up. Conclusion Only a few studies have investigated the risk of PCSM following initial negative biopsies and all studies included patients before the era of MRI of the prostate. However, the studies point to the fact that the risk of PCSM is low following initial negative TRUS biopsies, and that the level of prostate-specific antigen before biopsies holds prognostic information. This may be considered when advising patients about the need for further diagnostic evaluation.

PROSPERO registration number CRD42019134548.

\section{INTRODUCTION}

Systematic transrectal ultrasound-guided biopsies (TRUS biopsies) of the prostate have been gold standard for prostate cancer (PCa) detection since 1989 when Hodge et aldemonstrated that the sextant biopsy technique detected 9\% more cancers than targeted
Strengths and limitations of this study

- A total of 126672 men from four large populationbased studies were included.

- High quality of included studies.

- Main limitations were limited number of included studies, length of follow-up and missing information on risk factors for subgroups.

TRUS biopsies from hypoechoic areas in men with abnormal digital rectal examination. ${ }^{1}$ Sextant biopsies were routinely used until early 2000s where studies showed an increase of $19 \%-50 \%$ in PCa detection rates on TRUS biopsies by adding four cores from the lateral peripheral zone. ${ }^{2-5}$ This 10 -core biopsy scheme is considered standard procedure. ${ }^{3}$ In the past decades, improved treatment modalities and prostate-specific antigen (PSA) testing have fuelled the interest in early and accurate diagnosis of $\mathrm{PCa}^{6}$ Today, PCa has become the most frequently diagnosed cancer among men in the Western world. ${ }^{7}$ Undoubtedly, the increment in incidence of PCa worldwide is primarily a result of more frequent use of PSA as an early test for the disease in asymptomatic men, which has also led to increased diagnostic activity with TRUS biopsies. ${ }^{8}$ More men undergo systematic TRUS biopsies where biopsies turn out to be without cancer, that is, negative. The reported rate of false-negative systematic TRUS biopsies is $10 \%-34 \%$, but the clinical significance of these missed cancers is not well described. Many may be low grade, low volume and consequently of low risk to the patient. ${ }^{9-11}$ Moreover, the term clinical significance may have several definitions or endpoints. Recently, clinical significance in PCa has emerged as a pathological definition rather than a prognostic endpoint such as death from the disease. Clinical significance, 
as a prognostic definition in men with initial negative systematic TRUS biopsies, is not well studied.

Recent advancements in technology, such as multiparametric MRI (mpMRI) of the prostate, have led to an increased understanding of how and where systematic TRUS biopsies miss cancers and question the future role of this diagnostic strategy. ${ }^{12}$ All MRI studies define clinical significance according to prostate biopsy histology. Several studies have questioned the accuracy of PSA and TRUS biopsies in diagnosing clinically significant $\mathrm{PCa}$ (csPCa), especially in the re-biopsy setting in men with initial negative systematic TRUS biopsies. Clinically significance as a histological concept was originally introduced by Epstein $e t$ al as a tumour with a volume $>0.2 \mathrm{~cm}^{3}$, presence of Gleason pattern 4 or 5 , and a PSA density $>0.15 \mu \mathrm{g} / \mathrm{L} / \mathrm{g} .{ }^{13}$ Few studies use this definition strictly. MRI studies have shown that when mpMRI and targeted biopsy techniques are used in a re-biopsy setting in men with initial negative systematic TRUS biopsies, up to $50 \%$ are subsequently diagnosed with csPCa, defined by biopsy histology. ${ }^{14} 15$

Optimistically, the increased sensitivity results in more csPCa being diagnosed and because of an improved specificity, the rate of insignificant PCa (and thereby overdiagnosis) is reduced. ${ }^{16}{ }^{17}$ Rapidly, guidelines have recommended that diagnostic work-up for PCa include use of mpMRI both pre-biopsy and before re-biopsy after initial negative systematic TRUS biopsies. However, there is a potential gap between the histological definition of csPCa and prognosis. It remains questionable if all men with initial negative TRUS biopsies need MRI, or men can be safely omitted for further follow-up after negative TRUS biopsies. This paper systematically reviews the current evidence on the prognostic role of initial negative TRUS biopsies.

\section{METHODS}

This review was registered in Prospero (registration number CRD42019134548) and followed the Preferred Reporting Items for Systematic Reviews and Meta-Analyses guidelines $^{18}$ (see online supplemental appendix). Studies included should be randomised controlled trials or population-based cohort studies including men with initial negative TRUS biopsies and should present data on prostate cancer-specific mortality (PCSM). Studies reported in English in peer-reviewed journals from 1990 (when the use of TRUS biopsies was introduced) until present were included.

Searches were performed in PubMed and Embase databases using a search string combination with both keywords/Medical Subject Headings terms and free text in the advanced search builder (see online supplemental appendix for the full search string). Primary search string included:

1. Prostatic Neoplasm OR prostate cancer

2. Biopsy OR Image-guided biopsy OR transrectal ultrasound biopsy
3. Negative prostate biopsy OR benign initial

4. 1 AND 2 AND 3

Time of search was 13 April 2020. Based on titles, all relevant abstracts were screened independently by two authors (SMK, SBL) using Covidence, an online systematic review program, to identify studies that potentially met the described inclusion criteria. The selected full-text articles were evaluated by the two authors (SMK, SBL) for eligibility. Any disagreement was resolved by discussion with a third author (MAR). Data extraction was done using a predefined form by the same two authors independently and compared with confirm data.

To evaluate the quality of the included studies, two authors (SMK, SBL) independently and blindly to each other used the Newcastle-Ottawa Scale (NOS) for cohort studies when applicable (table 1). Risk of bias across studies was not assessed.

\section{Patient and public involvement}

No patient involvement.

\section{RESULTS}

In all, 523 records were identified, hereof 42 duplicates. Among the remaining 481 records screened by titles or abstract, 9 records were deemed relevant to the review. All records were assessed for eligibility, and five were excluded as they did not meet the inclusion criteria; either being a conference abstract only or with noneligible outcome or design. A flow chart for the inclusion of studies is shown in figure 1 .

Two of the included studies originated from North America ${ }^{1920}$ and two from Europe ${ }^{921}$ (see extracted information in table 2). One from each continent was based on a screening trial, the Rotterdam cohort of the European Randomized Study of Screening for Prostate Cancer $(\text { ERSPC })^{9}$ and the American Prostate, Lung, Colorectal and Ovarian Cancer Screening Trial. ${ }^{19}$ The two remaining studies originated from Canada and Denmark and were based on registry data. ${ }^{20}$ In total, the studies included 126672 men with initial negative TRUS biopsies.

The focus of this review was to determine PCSM reported on men with initial negative TRUS biopsies in the included studies.

\section{Cancer-specific mortality}

The risk of subsequent PCSM among men with initial negative TRUS biopsies ranged from $0.03 \%$ to $2 \%$ in the studies with a median follow-up ranging from 5.9 to 11 years (see table 2 ).

Three of the included studies calculated the cumulative incidence of PCSM. ${ }^{19-21}$ The 20-year cumulative incidence of PCSM was $5.2 \%$ (95\% CI $3.9 \%$ to $6.5 \%$ ) in the Danish study by Klemann et al. ${ }^{21}$ Of special interest, the study demonstrated that in men with PSA $<10 \mu \mathrm{g} / \mathrm{L}$ prior to negative TRUS biopsies, the cumulative incidence of PCSM at 15 years was only $0.7 \%$ (95\% CI $0.2 \%$ to $1.3 \%$ ). The median follow-up time was 5.9 years (IQR 3.8-8.5) 
Table 1 Newcastle-Ottawa Scale for quality assessment of a cohort study, maximum score 9

\begin{tabular}{|c|c|c|c|c|c|}
\hline \multicolumn{2}{|l|}{ Selection } & \multicolumn{2}{|l|}{ Comparability } & \multicolumn{2}{|l|}{ Outcome } \\
\hline \multicolumn{2}{|l|}{ Selection of intervention cohort } & \multicolumn{2}{|c|}{$\begin{array}{l}\text { Comparability of cohorts on basis of design } \\
\text { or analysis }\end{array}$} & \multicolumn{2}{|l|}{ Assessment of outcome } \\
\hline Somewhat representative & 1 & & & Record linkage & 1 \\
\hline Selected group of patients & 0 & & & Self-report & 0 \\
\hline \multicolumn{2}{|c|}{ Selection of non-intervention cohort } & $\begin{array}{l}\text { Study control for any additional } \\
\text { factors }\end{array}$ & 1 & \multicolumn{2}{|l|}{$\begin{array}{l}\text { Was follow-up long enough for } \\
\text { outcome to occur? }\end{array}$} \\
\hline $\begin{array}{l}\text { Same community as } \\
\text { intervention cohort }\end{array}$ & 1 & & & \multirow[t]{2}{*}{$\begin{array}{l}\text { Yes, if median duration of } \\
\text { follow-up }>5 \text { years }\end{array}$} & 1 \\
\hline From different source & 0 & & & & \\
\hline Secure record & 1 & & & \multirow{4}{*}{\multicolumn{2}{|c|}{ Adequacy of follow-up of cohorts }} \\
\hline Structured interview & 1 & & & & \\
\hline Written self-report & 0 & & & & \\
\hline Other/no description & 0 & & & & \\
\hline \multicolumn{2}{|c|}{$\begin{array}{l}\text { Demonstration that outcome of interest } \\
\text { was not present at start of study }\end{array}$} & & & Complete follow-up & 1 \\
\hline Yes & 1 & & & \multirow{2}{*}{$\begin{array}{l}\text { Subjects lost to follow-up, } \\
<20 \%\end{array}$} & \multirow[t]{2}{*}{1} \\
\hline \multirow[t]{2}{*}{ No } & 0 & & & & \\
\hline & & & & Follow-up <80\% & 0 \\
\hline
\end{tabular}

but a large number of men had more than 15 years of follow-up. The 20-year cumulative incidence of PCSM was $1.8 \%(95 \%$ CI $1.6 \%$ to $2.0 \%)$ in the Canadian study by Sayyid et $a .^{20}$ The American study by Lewicki et $a l^{19}$ found an 11-year cumulative incidence of PCSM of about $1.8 \%$ (95\% CI not reported). Among men with initial negative TRUS biopsies in the ERSPC trial, Schröder et al reported a $3 \%$ risk of $\mathrm{PCa}$ death at 11 years. ${ }^{9}$

\section{Quality of studies}

None of the included studies presents a description of the derivation of their cohort including more detailed information on patient inclusion and exclusion apart from 'no prior diagnosis of PCa', and risk of bias due to patient selection can therefore not be eliminated. Two of the studies ${ }^{9}{ }^{19}$ were based on trials from which the inclusion and exclusion information could be retrieved. ${ }^{22} 23$ The cohorts were from a secure record indicating low risk of bias, and the assessment of outcome was all linked to a record except for the cohort in the American study by Lewicki et $a l,{ }^{19}$ which was based on self-reports presenting intermediate risk of bias. The study by Klemann et $a t^{21}$ stated a complete follow-up. The other studies did not state their loss to follow-up. For the studies, the quality scores on the NOS ranged from 5 to 7 , but for one study the NOS was non-applicable and therefore a minimum score is stated (see table 3 ). Risk of publication bias has not been assessed.

\section{DISCUSSION}

We identified four studies where risks of PCSM following initial negative TRUS biopsies have been reported. Despite interstudy differences, differences in other-cause mortality, follow-up time and re-biopsy strategies, the studies find an almost identical low risk of subsequent PCSM at 10-20 years following initial negative systematic TRUS biopsies.

Two of the studies originated from randomised trials investigating the effect of systematic PSA-based screening to reduce the risk of PCSM and thus the primary endpoint was not to investigate the cohort of men in which the TRUS biopsies were negative. Furthermore, differences in pre-biopsy strategies may affect PCSM. Men in the Canadian and Danish population did not undergo systematic PSA testing, and the median PSA from the American screening trial was $4.89 \mu \mathrm{g} / \mathrm{L}$ (IQR 1.88-6.36) ${ }^{19}$ and the median PSA from a non-screened population-based cohort was $7.7 \mu \mathrm{g} / \mathrm{L}(\mathrm{IQR} 5.5-12.0){ }^{21}$ 


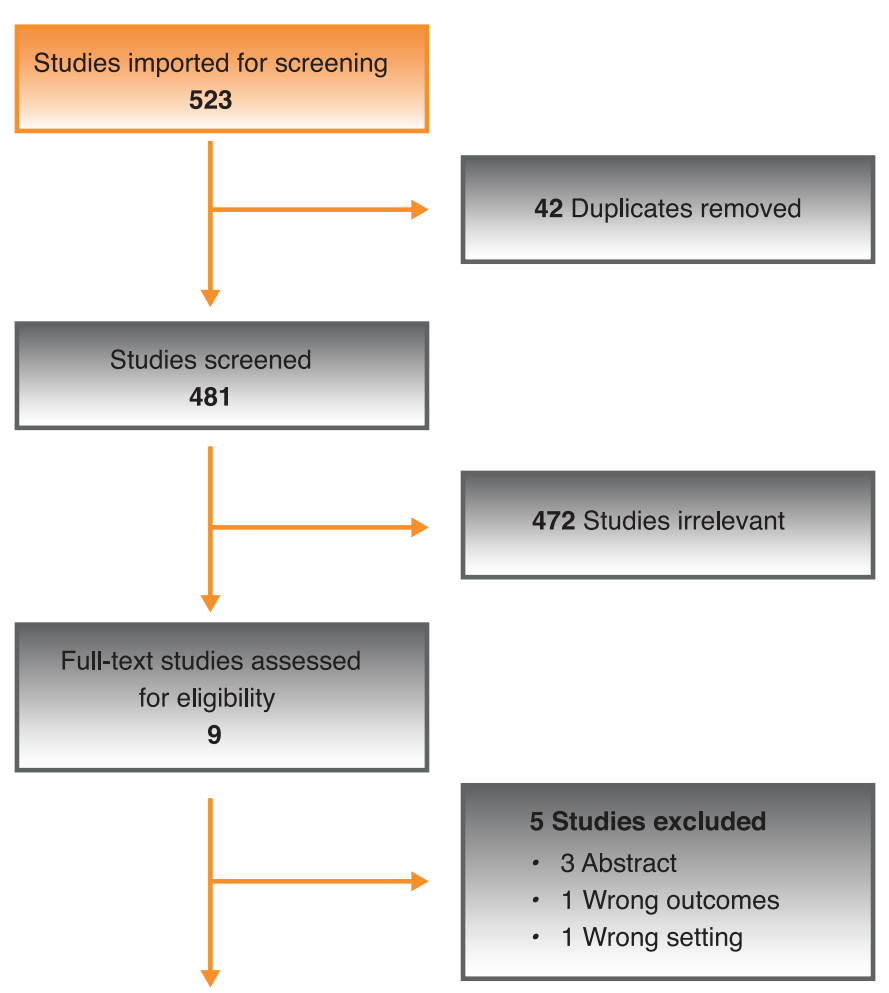

Studies included

4

Figure 1 Flow diagram of the process used to select studies for the systematic review. We systematically searched PubMed and Embase.

The Danish study provided PSA at initial biopsies in $15 \%$ of the men with benign initial biopsies and confirmed the prognostic value of PSA as men with low PSA at the initial biopsies had the lowest risk of subsequent PCSM. ${ }^{21}$ The Canadian study stratified results by age and found age as a significant predictor of PCSM. ${ }^{20}$ Age and length of follow-up will affect the estimated risk of PCSM in the four included studies, why any comparison should be interpreted with caution. The Canadian study has the widest age span and included all men aged 40 years and older. ${ }^{20}$ Since younger men are more likely to die of PCa and old men are at high risk of dying from something else, this might have affected the risk of PCSM. However, the median age in the Canadian study is comparable with the American study. ${ }^{19}$ Men included in the Danish study were slightly older ${ }^{21}$ whereas the Dutch study only states the age intervals. ${ }^{9}$ Duration of follow-up varies between the four studies, and it is not clear how patients with initial negative TRUS biopsies were managed over time, for example, when, why and how often patients were offered re-biopsies. Logically, such interstudy differences may affect detection, incidence and likelihood of subsequent treatment which ultimately may affect PCSM.

Overall, the studies lacked information on risk groups in which lethal cancers potentially were missed. The American study was mainly based on questionnaires, which increases the risk of loss to follow-up. ${ }^{19}$ The Dutch,
Danish and Canadian studies ${ }^{92} 21$ were register-based which introduce a risk of bias due to the inherent risk of misclassification of the cause of death, correct diagnosis and so on. All studies included information on the risk of bias for the cause of death. The Danish Cause of Death Registry has been reported to have $>93 \%$ concordance between the cause of death registered in the registry and cause of death based on information from patient files. ${ }^{24}$ PCSM as the outcome was retrieved from a national registry in the Danish study ${ }^{21}$ and in the screening trials, this was evaluated by a cause-of-death committee for each death. ${ }^{9}{ }^{19}$ In the American study, deaths were confirmed from death certificates. ${ }^{19}$

During the past decade, an intense interest in the use of MRI and MRI-TRUS fusion targeted biopsies to diagnose and to reduce the risk of missing csPCa has evolved-also in men with initial negative systematic TRUS biopsies. The potential to diagnose more csPCa and at the same time keep the detection of insignificant PCa low has led to a large number of studies on the subject.

A systematic review by Fütterer $e t$ al showed that the use of MRI-targeted biopsies found a median of $50 \%$ (IQR $48 \%-53 \%$ ) csPCa in men aged $60-80$ years with previous negative biopsies. ${ }^{25}$ The included studies in the review used histology of TRUS biopsies as the reference standard. The very high prevalence of csPCa is noteworthy as it is higher than what is reported in a recent systematic review of PCa in autopsies, where the prevalence of autopsydetected PCa in the same age group was $30 \%-40 \% .{ }^{26}$ However, caution is called for when comparing prevalence of PCa in autopsy studies with prevalence in biopsy materials, respectively, as several biases with selection as the most obvious, may play a role. In the papers included in the review by Fütterer $e t a l$, various definitions of csPCa were used including different biopsy Gleason scores (GS), number and length of positive cores and PSA levels. ${ }^{25}$ The studies primarily included re-biopsies in men with positive mpMRIs and disregarded the negative mpMRIs which could bias the true negative and false negative values. Most of the included studies used radical prostatectomy specimen as the reference standard, which also represents an important bias as only MRI-positive men went on to undergo surgery.

A recent Cochrane review by Drost et al demonstrated that csPCa (GS $3+4$ or higher) was found in $22.8 \%(95 \%$ CI $20.0 \%$ to $26.2 \%$ ) of men with initial negative biopsies in a re-biopsy setting with a combination of both an MRI pathway and TRUS biopsies using template biopsies as the reference standard. ${ }^{27}$ The differences in detection of csPCa in the reviews by Fütterer $e t a l$ and Drost $e t a l$, respectively, are probably caused by the different reference standards. Template biopsies are more likely to represent the true pathology of the prostate than systematic TRUS biopsies. Drost $e t$ al found the detection ratio of MRI pathway versus TRUS biopsies in men with initial negative TRUS biopsies to be 1.44 (95\% CI 1.19 to 1.75 ). In men with initial negative TRUS biopsies and a negative mpMRI, Drost et al also showed that 5.3\% (95\% CI 


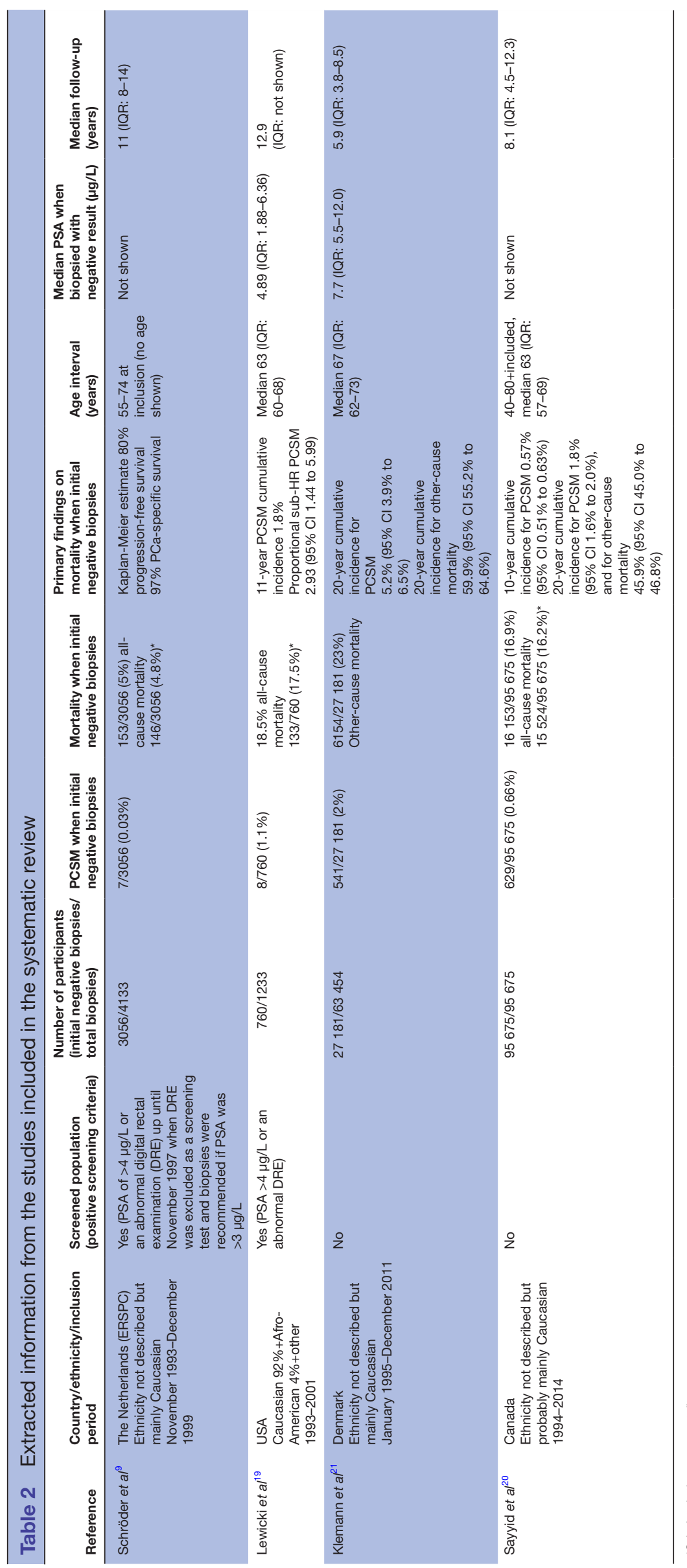

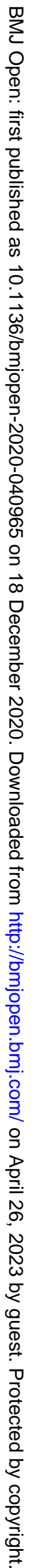


Table 3 Newcastle-Ottawa Scale (NOS), risk of bias assessment

\begin{tabular}{|c|c|c|c|c|}
\hline Assessment of quality of studies & $\begin{array}{l}\text { Selection } \\
(0-4)\end{array}$ & $\begin{array}{l}\text { Comparability } \\
(0-2)\end{array}$ & $\begin{array}{l}\text { Outcome } \\
(0-3)\end{array}$ & $\begin{array}{l}\text { Total } \\
(0-9)\end{array}$ \\
\hline Schröder et al ${ }^{9}$ & 3 & 0 & 2 & 5 \\
\hline Klemann et $a^{21}$ & 3 & 1 & 3 & 7 \\
\hline Sayyid et al ${ }^{20 *}$ & 2 & 2 & 2 & 6 \\
\hline
\end{tabular}

*No non-intervention cohort in the study why NOS is non-applicable to assess the real quality of the study.

$3.1 \%$ to $8.9 \%$ ) had csPCa when systematic biopsies were applied despite a negative MRI but on the cost of $14.2 \%$ (95\% CI $5.9 \%$ to $30.2 \%$ ) detected with insignificant PCa.

Overall, MRI studies indicate that $20 \%-50 \%$ of men with initial negative TRUS biopsies harbour csPCa defined as GS $3+4$ or higher. Our review underlines the marked difference between a histological definition of csPCa at re-biopsy and csPCa defined as lethal disease in men with initial negative systematic TRUS biopsies. Recent studies have found that men with negative mpMRI of the prostate hold little risk of harbouring csPCa. It can be speculated whether in the future, negative mpMRI will hold the same prognostic information as negative TRUS biopsies. However, mpMRI may miss some potentially csPCa as in the case of TRUS biopsies. ${ }^{28} 29$

\section{LIMITATIONS}

Only a few studies have investigated the risk of PCSM in men with initial negative biopsies which is the most important limitation of this systematic review. We fully acknowledge the limitation of searching only two databases aswell. Second, the included studies to a large extent lacked information on pre-biopsy PSA or other prognostic parameters which is important if these data should be translated into clinical decisions. The lack of data also impaired the possibility to perform a metaanalysis which could have been beneficial for the review. Lastly, the short follow-up in all studies is an overall limitation that entails a careful interpretation of the results. Small aggressive tumours missed by TRUS biopsies may evolve into a lethal disease after a long time. Thus, length of follow-up is essential for evaluating the risk of PCSM.

\section{Results in context}

The results presented emerge from patients that may not resemble contemporary patients. The diagnostic strategy has changed thus it should be debated if the estimates from the included cohorts are representative in a modern context. Increasing the number of prostate biopsies and the introduction of image-guided biopsies have been demonstrated to increase the number of cancers diagnosed. Furthermore, more men are subjected to systematic or opportunistic PSA testing potentially lowering the threshold for diagnosis. These changes may reinforce that contemporary men with initial negative TRUS biopsies have an even lower risk of PCSM.
The optimal management of these men has spurred controversy, ranging from nothing to PSA follow-up to TRUS-guided re-biopsies and/or mpMRI. The present structured review adds to the discussion by demonstrating that initial negative systematic TRUS biopsies hold strong prognostic information which is amplified further by the addition of PSA.

The role of MRI in the follow-up in men with initial negative TRUS biopsies should be discussed. Currently, published papers on MRI and targeted biopsies compared with standard systematic TRUS biopsies conclude on the assumption that the cancers detected by targeted biopsies are of the same biological nature, but this may be far from the truth, as addressed by Vickers $e t$ al in a recent editorial. ${ }^{30}$ Our review underlines that few lethal PCa are missed at the initial TRUS biopsies and questions the definition of csPCa on targeted MRI-guided biopsies. No study has previously clarified the true effect of the MRI pathway in a randomised setting for men with initial negative TRUS biopsies comparing mpMRI and targeted biopsies versus systematic TRUS biopsies in the re-biopsy setting. Also, the clinical implication of csPCa detected with the MRI pathway needs to be addressed by studies with clinical endpoints such as PSA progression, metastases and PCSM.

\section{CONCLUSION}

Clinically significant PCa, defined as a lethal disease, is rarely missed in the initial systematic TRUS biopsies. In the continuing struggle to reduce overdiagnosis, it remains important to define the optimal candidate for re-biopsy when the initial biopsies are negative. The follow-up strategy and use of mpMRI in men with initial negative biopsies need further evaluation in randomised controlled trials.

Contributors SMK designed the systematic review protocol, with support of SBL and MAR. SMK prepared the first draft. SBL, MAR and KB reviewed and revised the first draft. SMK, SBL, JTH, PI, MAR and KB revised later drafts, read and approved the final manuscript.

Funding The authors have not declared a specific grant for this research from any funding agency in the public, commercial or not-for-profit sectors.

Competing interests None declared.

Patient consent for publication Not required.

Provenance and peer review Not commissioned; externally peer reviewed. 
Data availability statement All data relevant to the study are included in the article or uploaded as supplemental information. No additional data available.

Supplemental material This content has been supplied by the author(s). It has not been vetted by BMJ Publishing Group Limited (BMJ) and may not have been peer-reviewed. Any opinions or recommendations discussed are solely those of the author(s) and are not endorsed by BMJ. BMJ disclaims all liability and responsibility arising from any reliance placed on the content. Where the content includes any translated material, BMJ does not warrant the accuracy and reliability of the translations (including but not limited to local regulations, clinical guidelines, terminology, drug names and drug dosages), and is not responsible for any error and/or omissions arising from translation and adaptation or otherwise.

Open access This is an open access article distributed in accordance with the Creative Commons Attribution Non Commercial (CC BY-NC 4.0) license, which permits others to distribute, remix, adapt, build upon this work non-commercially, and license their derivative works on different terms, provided the original work is properly cited, appropriate credit is given, any changes made indicated, and the use is non-commercial. See: http://creativecommons.org/licenses/by-nc/4.0/.

\section{ORCID iDs}

Sandra Miriam Kawa http://orcid.org/0000-0001-9162-0501

Signe Benzon Larsen http://orcid.org/0000-0001-9522-7678

John Thomas Helgstrand http://orcid.org/0000-0001-6724-6003

Klaus Brasso http://orcid.org/0000-0001-6562-6416

Martin Andreas Røder http://orcid.org/0000-0002-0019-5333

\section{REFERENCES}

1 Hodge KK, McNeal JE, Terris MK, et al. Random systematic versus directed ultrasound guided transrectal core biopsies of the prostate. $J$ Urol 1989;142:71-4.

2 Eskicorapci SY, Baydar DE, Akbal C, et al. An extended 10-core transrectal ultrasonography guided prostate biopsy protocol improves the detection of prostate cancer. Eur Urol 2004;45:444-9.

3 Stamatiou K, Alevizos A, Karanasiou V, et al. Impact of additional sampling in the TRUS-guided biopsy for the diagnosis of prostate cancer. Urol Int 2007:78:313-7.

4 Durkan GC, Sheikh N, Johnson P, et al. Improving prostate cancer detection with an extended-core transrectal ultrasonography-guided prostate biopsy protocol. BJU Int 2002;89:33-9.

5 Al-Ghazo MA, Ghalayini IF, Matalka II. Ultrasound-Guided transrectal extended prostate biopsy: a prospective study. Asian J Androl 2005;7:165-9.

6 Ellis WJ, Chetner MP, Preston SD, et al. Diagnosis of prostatic carcinoma: the yield of serum prostate specific antigen, digital rectal examination and transrectal ultrasonography. $J$ Urol 1994;152:1520-5.

7 Bray F, Ferlay J, Soerjomataram I, et al. Global cancer statistics 2018: GLOBOCAN estimates of incidence and mortality worldwide for 36 cancers in 185 countries. CA Cancer J Clin 2018;68:394-424.

8 Helgstrand JT, Klemann N, Røder MA, et al. Danish Prostate Cancer Registry - methodology and early results from a novel national database. Clin Epidemiol 2016;8:351-60.

9 Schröder FH, van den Bergh RCN, Wolters T, et al. ElevenYear outcome of patients with prostate cancers diagnosed during screening after initial negative sextant biopsies. Eur Urol 2010;57:256-66.

10 Tan N, Lane BR, Li J, et al. Prostate cancers diagnosed at repeat biopsy are smaller and less likely to be high grade. J Urol 2008;180:1325-9.

11 Djavan B, Mazal P, Zlotta A, et al. Pathological features of prostate cancer detected on initial and repeat prostate biopsy: results of the prospective European prostate cancer detection study. Prostate 2001;47:111-7.

12 Boesen L, Nørgaard N, Løgager V, et al. Where do transrectal Ultrasound- and magnetic resonance imaging-guided biopsies miss significant prostate cancer? Urology 2017;110:154-60.

13 Epstein JI, Walsh PC, Carmichael M. Pathologic and clinical findings to predict tumor extent of nonpalpable (stage T1 C) prostate cancer. JAMA 1994;271:368

14 Schouten DI FFG, van der Leest M, Pokorny M, et al. Prostate cancer why and where do we miss significant prostate cancer with multi-parametric magnetic resonance imaging followed by magnetic resonance-guided and transrectal ultrasound-guided biopsy in Biopsy-naïve men? Eur Urol 2016;8:6-9.

15 Lee SH, Chung MS, Kim JH, et al. Magnetic resonance imaging targeted biopsy in men with previously negative prostate biopsy results. J Endourol 2012;26:787-91.

16 Barentsz JO, Richenberg J, Clements R, et al. ESUR prostate Mr guidelines 2012. Eur Radiol 2012;22:746-57.

17 Boesen L, Thomsen HS. [Magnetic resonance imaging facilitate appropriate treatment selection of prostate cancer patients]. Ugeskr Laeger 2013;175:1634-7.

18 Moher D, Liberati A, Tetzlaff J, et al. Preferred reporting items for systematic reviews and meta-analyses: the PRISMA statement. Int $J$ Surg 2010;8:336-41.

19 Lewicki P, Shoag J, Golombos DM, et al. Prognostic significance of a negative prostate biopsy: an analysis of subjects enrolled in a prostate cancer screening trial. Journal of Urology 2017;197:1014-9.

20 Sayyid RK, Alibhai SMH, Sutradhar R, et al. Population-Based outcomes of men with a single negative prostate biopsy: importance of continued follow-up among older patients. Urol Oncol 2019;37:19-27

21 Klemann N, Røder MA, Helgstrand JT, et al. Risk of prostate cancer diagnosis and mortality in men with a benign initial transrectal ultrasound-guided biopsy set: a population-based study. Lancet Oncol 2017:18:221-9.

22 Schröder $\mathrm{FH}$, Bangma $\mathrm{CH}$. The European randomized study of screening for prostate cancer (ERSPC). Br J Urol 1997;79 Suppl 1:68-71.

23 Prorok PC, Andriole GL, Bresalier RS. Design of the prostate, lung, colorectal and ovarian (PLCO) cancer screening trial. New York, NY, 2000: 273S-309.

24 Larsen SB, Brasso K, Christensen J, et al. Socioeconomic position and mortality among patients with prostate cancer: influence of mediating factors. Acta Oncol 2017;56:563-8.

25 Fütterer JJ, Briganti A, De Visschere P, et al. Can clinically significant prostate cancer be detected with multiparametric magnetic resonance imaging? A systematic review of the literature. Eur Urol 2015;68:1045-53.

26 Bell KJL, Del Mar C, Wright G, et al. Prevalence of incidental prostate cancer: a systematic review of autopsy studies. Int $\mathrm{J}$ Cancer 2015; 137:1749-57.

27 Drost F-JH, Osses D, Nieboer D, et al. Prostate magnetic resonance imaging, with or without magnetic resonance Imaging-targeted biopsy, and systematic biopsy for detecting prostate cancer: a Cochrane systematic review and meta-analysis. Eur Urol 2020;77:7894.

28 Panebianco V, Barchetti G, Simone G, et al. Negative multiparametric magnetic resonance imaging for prostate cancer: what's next? Eur Urol 2018;74:48-54.

29 Ahdoot M, Wilbur AR, Reese SE, et al. MRI-Targeted, systematic, and combined biopsy for prostate cancer diagnosis. N Engl J Med 2020;382:917-28.

30 Vickers A, Carlsson SV, Cooperberg M, et al. Routine use of magnetic resonance imaging for early detection of prostate cancer is not justified by the clinical trial evidence. Eur Urol 2020;78:304-6. 